\title{
Progress in the Treatment of Neuroendocrine Tumors
}

\author{
Jennifer A. Chan, MD, MPH and Matthew H. Kulke, MD, MMSc
}

\begin{abstract}
Traditional treatments for patients with advanced neuroendocrine tumors include surgical debulking, hepatic embolization, somatostatin analogues, and interferon-a. Patients with pancreatic neuroendocrine tumors also may benefit from treatment with the alkylating agents streptozocin or temozolomide. In recent years, several promising new approaches have been investigated in patients with advanced neuroendocrine tumors. One such approach has been the use of radiopeptide therapy targeting somatostatin receptors. Additionally, agents targeting the vascular endothelial growth factor pathway and mammalian target of rapamycin have shown preliminary evidence of activity and are currently being evaluated in large randomized studies.
\end{abstract}

\section{Introduction}

Most neuroendocrine tumors can be subclassified into two general classes: carcinoid tumors and pancreatic neuroendocrine tumors. Tumors originating from the gastrointestinal tract, lung, and thymus traditionally are defined as carcinoid tumors, whereas those arising in the pancreas or peripancreatic region are commonly classified as pancreatic neuroendocrine tumors. The annual incidence of clinically relevant carcinoid tumors previously was estimated to be one to two per 100,000 population per year [1]. However, recent studies suggest that the annual incidence of carcinoid tumors may be higher. In a recent analysis of the Surveillance, Epidemiology and End Results (SEER) database, the estimated annual incidence of carcinoid tumors was 5.25 per 100,000 population, and the limited duration prevalence in the United States was estimated to exceed 100,000 individuals [2•]. These increases in diagnosed incidence and prevalence are likely attributable, in part, to the increasing awareness and improved diagnostic strategies for neuroendocrine tumors.

Because of the heterogeneity in morphology and clinical behavior of neuroendocrine tumors, the World Health Organization updated a classification system for these tumors in 2000 [3]. In this system, a distinction is made between well-differentiated neuroendocrine tumors, which are characterized by indolent behavior; well-differentiated neuroendocrine carcinomas, which pursue an intermediate clinical course; and poorly differentiated neuroendocrine carcinomas, which are characterized by an aggressive clinical course and a poor prognosis. The terms carcinoid and pancreatic endocrine tumor generally refer to well-

Copyright $\odot 2009$ by Current Medicine Group LLC

Corresponding author. Jennifer A. Chan, MD, MPH, Gastrointestinal Cancer Center, Dana-Farber Cancer Institute, 44 Binney

Street, Boston, MA 02115, USA., jang@ partners.org.

Disclosure

No potential conflicts of interest relevant to this article were reported. 
differentiated neuroendocrine tumors. These well-differentiated tumors also are most commonly associated with symptoms of hormonal hypersecretion. The secretion of serotonin and other vasoactive substances causes the carcinoid syndrome, which is manifested by episodic flushing, wheezing, diarrhea, and eventual right-sided valvular heart disease [4]. Syndromes associated with hormone-secreting pancreatic neuroendocrine tumors include those associated with insulinoma, glucagonoma, vasoactive intestinal peptide (VIP)-oma, and gastrinoma.

The treatment of patients with localized neuroendocrine tumors primarily is surgical. There currently are no data to suggest a benefit associated with postoperative adjuvant therapy. The treatment of patients with metastatic neuroendocrine tumors usually is based on an assessment of symptoms, performance status, disease burden, and rates of disease progression. Patients with indolent, asymptomatic metastatic disease occasionally are followed up without treatment until there is evidence of progression or until symptoms develop. Patients with symptoms or evidence of disease progression are considered for a broad range of treatment options.

\section{Surgical Treatment of Metastatic Disease}

In selected cases, metastatic liver disease can be surgically resected. In one large surgical series involving 170 patients undergoing hepatic resection, more than $90 \%$ achieved improvement in symptoms. Although the recurrence rate following surgery was high (84\%), the 5-year and 10-year survival rates were encouraging (61\% and 35\%, respectively) [5]. A large number of liver metastases may preclude hepatic resection. In this instance, the issue of hepatic transplantation may be raised. The number of patients with liver-isolated metastatic disease in whom orthotopic liver transplantation (OLT) has been attempted is small, and the role of OLT in such patients remains unclear [6-8]. Early studies reported high rates of both perioperative mortality and tumor recurrence. Results from a recent multicenter study are more encouraging, demonstrating a 5-year overall survival rate of $69 \%$ for patients with carcinoid tumors [8]. Nevertheless, the impact of transplantation on the natural history of disease in these patients is difficult to assess because selected patients may have indolent disease regardless of the therapeutic approach. The lack of available transplants also precludes OLT as a treatment option in many locations.

\section{Hepatic Artery Embolization}

In contrast to OLT, hepatic artery embolization is commonly used as a palliative technique in patients who have hepatic metastases but are not candidates for surgical resection.

Hepatic artery embolization is based on the principle that tumors in the liver derive most of their blood supply from the hepatic artery, whereas healthy hepatocytes derive most of their blood supply from the portal vein. Embolization response rates are measured by a decrease in hormonal secretion or by radiographic regression and are generally greater than $50 \%$ $[9,10]$. However, the duration of response may be brief, ranging from 4 to 51 months in uncontrolled patient series $[9,11]$. In one of the largest series, in 81 patients undergoing embolization or chemoembolization for carcinoid tumors, the median duration of response was 17 months, and the probability of progression-free survival at 1,2, and 3 years was 
$75 \%, 35 \%$, and $11 \%$, respectively [9]. Early studies reported a significant incidence of postembolization complications including renal failure, hepatic necrosis, and sepsis. However, in recent years, improved techniques have reduced the incidence of such complications, making embolization an important and generally safe treatment option for patients with neuroendocrine tumors.

\section{Somatostatin Analogues}

The carcinoid syndrome, as well as other hormonal syndromes associated with neuroendocrine tumors, often can be well controlled with somatostatin analogues. Somatostatin is a 14-amino acid peptide that inhibits the secretion of a broad range of hormones and acts by binding to somatostatin receptors, which are expressed on most neuroendocrine tumors [12]. In an initial study, the subcutaneous administration of the somatostatin analogue octreotide, administered at a dosage of $150 \mu \mathrm{g}$ three times a day, improved the symptoms of carcinoid syndrome in $88 \%$ of patients [13]. More recently, the use of a long-acting depot form of octreotide, which can be administered on a monthly basis, largely has obviated the need for patients to inject themselves on a daily basis. Long-acting octreotide is typically initiated at a dose of $20 \mathrm{mg}$ intramuscularly after a brief trial of the short-acting formulation, with gradual escalation of the dose as needed for optimal control of symptoms [14]. Patients also may use additional short-acting octreotide for break-through symptoms.

Lanreotide, another somatostatin analogue, appears to be similar to octreotide in its clinical efficacy for carcinoid syndrome, and can be self-administered as a long-acting subcutaneous injection. A randomized study of lanreotide SR (slow release) versus octreotide in 33 patients with carcinoid syndrome demonstrated similar rates of symptom control and reduction of biochemical markers [14].

Novel somatostatin analogues that are more broadly targeted and have higher affinities for somatostatin receptors have been developed recently. Pasireotide (SOM230) is a multiligand somatostatin analogue that has exhibited high binding affinity to sst1, sst2, sst3, and sst5. Compared with octreotide, pasireotide has 30, 5, and 40 times more binding affinity for sst1, sst3, and sst5 receptors, respectively, and comparable affinity for sst2 [15]. In a phase 2 trial, 44 patients with metastatic carcinoid tumors whose symptoms of diarrhea and flushing were inadequately controlled by octreotide LAR (long-acting release) received pasireotide, $300 \mu \mathrm{g}$ subcutaneously twice per day and escalated to a maximum dose of $1200 \mu \mathrm{g}$ twice per day every 3 days until symptom control was achieved. Control of symptoms was achieved in 12 of 44 patients $(27 \%)$ [16].

\section{Interferon- $a$}

The addition of interferon (IFN)- $a$ to therapy with somatostatin analogues was reported to be effective in controlling symptoms in patients with the carcinoid syndrome who may be resistant to somatostatin analogues alone $[17,18]$. Therapy with low-dose IFN-a subsequently was reported to result in biochemical responses in approximately $40 \%$ of patients with metastatic neuroendocrine tumors, and is occasionally associated with tumor regression [19]. In clinical trials, doses of IFN-a have ranged from 3 to $9 \mathrm{MU}$ 
subcutaneously, administered three to seven times per week. IFN-a is somewhat myelosuppressive, and the dose often is titrated in individual patients to achieve a total leukocyte count of 3000/ $\mu \mathrm{L}$. The more widespread acceptance of IFN-a in the treatment of metastatic neuroendocrine tumors has been limited by studies challenging its efficacy as well as the potential for side effects, which may include myelosuppression, fatigue, depression, and alteration of thyroid function.

\section{Cytotoxic Chemotherapy}

Although carcinoid and pancreatic neuroendocrine tumors appear histologically similar, there is increasing evidence that pancreatic neuroendocrine tumors are more responsive than carcinoid tumors to chemotherapy. In a recent trial, 249 patients with advanced carcinoid tumors were randomly assigned to receive streptozocin/5-fluorouracil (5-FU) or 5-FU/ doxorubicin [20]. The response rates associated with the two regimens were low but similar (16\% vs $15.9 \%)$. Although there was a slightly longer survival time associated with streptozocin/5-FU (24.3 vs 15.7 months) in this trial, more than one third of the patients treated with streptozocin developed renal toxicity.

In contrast, patients with pancreatic neuroendocrine tumors may respond well to treatment with streptozocin and other alkylating agents (Table 1). In an initial randomized trial, the combination of streptozocin and doxorubicin was associated with a combined biochemical and radiologic response rate of $69 \%$ together with a survival benefit, with a median overall survival of 2.2 years [21]. Streptozocin subsequently was approved by the US Food and Drug Administration as a treatment for patients with pancreatic neuroendocrine tumors. The very high reported response rates in this study have been questioned and are likely the result of the use of nonstandard response criteria. A retrospective analysis of 84 patients with locally advanced or metastatic pancreatic endocrine tumors receiving a three-drug regimen of streptozocin, 5-FU, and doxorubicin showed that this regimen was associated with an overall response rate of $39 \%$ and a median survival of 37 months [22].

Temozolomide is an orally available cytotoxic alkylating agent with a mechanism of action similar to that of streptozocin and dacarbazine [23]. In a prospective study, treatment with temozolomide and thalidomide was associated with objective responses in 5 of 11 patients (45\%) with pancreatic neuroendocrine tumors and 1 of 14 patients with carcinoid tumors [24]. In a second prospective study, treatment with temozolomide and bevacizumab was associated with tumor responses in 4 of 17 patients (24\%) with pancreatic neuroendocrine tumors but 0 of 12 patients with carcinoid tumors [25]. Both regimens incorporated a doseintense temozolomide regimen of $150 \mathrm{mg} / \mathrm{m}^{2}$ daily for 7 days, administered on an everyother-week schedule. Retrospective series further support the use of temozolomide in pancreatic neuroendocrine tumors. In a series of 36 patients treated with temozolomide monotherapy, tumor regression was observed in $31 \%$ of bronchial carcinoid tumors and $8 \%$ of pancreatic neuroendocrine tumors [26]. In other retrospective series, which included only patients with pancreatic neuroendocrine tumors, combination therapy with temozolomide and capecitabine was associated with tumor response rates of $59 \%$ to $71 \%[27,28]$. 


\section{Radiopeptide Therapy}

A high incidence of somatostatin receptor expression in neuroendocrine tumors provides the rationale for radionuclide therapy as a treatment modality for patients with inoperable or metastatic disease. Several radiolabeled somatostatin analogues have been developed to treat patients with somatostatin receptor-positive metastatic tumors. The available radiolabeled somatostatin analogues differ from one another in their affinity for the various somatostatin receptor subtypes and in the radionuclides to which they are conjugated [29]. The most frequently used radionuclides for targeted radiotherapy are indium $111\left({ }^{111} \mathrm{In}\right)$, yttrium 90 $\left({ }^{90} \mathrm{Y}\right)$, and lutetium $177\left({ }^{177} \mathrm{Lu}\right)$, which differ from one another in terms of emitted particles, particle energy, and tissue penetration [30,31].

${ }^{111}$ In-labeled octreotide, which emits $\beta$-radiation and Auger electrons, has been evaluated in several clinical studies. However, there are few reports of objective tumor responses [32]. Radionuclides emitting $\beta$-radiation may have better therapeutic advantage because the emitted particle ranges are longer [29]. Encouraging results have been obtained with various somatostatin analogues coupled to ${ }^{90} \mathrm{Y}$, a high-energy $\beta$-particle emitter. The first somatostatin analogue radiolabeled with ${ }^{90} \mathrm{Y}$ and used for peptide receptor radionuclide therapy was [DOTA,Tyr3]octreotide (DOTATOC). In comparison with octreotide, the phenylalanine residue at position 3 has been replaced with tyrosine, making the compound more hydrophilic, with increased affinity for the somatostatin receptor sst2 [29]. In phase 1 and 2 studies of ${ }^{90}$ Y-DOTATOC, objective radiologic responses were noted in up to $34 \%$ of patients with metastatic neuroendocrine tumors [33]. Another ${ }^{90} \mathrm{Y}$-labeled somatostatin analogue that has been used for peptide receptor radionuclide therapy is DOTA-lanreotide. This is a more lipophilic compound with a high affinity for sst5 and an affinity for sst2 comparable with that of octreotide [29]. In a multicenter European study of $\left[{ }^{90} \mathrm{Y}\right.$ DOTA]lanreotide ( ${ }^{90}$ Y-DOTALAN), $20 \%$ of patients with neuroendocrine tumors demonstrated objective responses to therapy [34]. The new somatostatin analogue [Tyr3] octreotate, which has very high affinity for sst2, differs from octreotide by a threonine residue, which replaces the C-terminal threonine in octreotide [29]. A study of patients with metastatic neuroendocrine tumors treated with ${ }^{90} \mathrm{Y}$-labeled [DOTA,Tyr3] octreotate $\left({ }^{90} \mathrm{Y}\right.$ DOTATATE) reported objective responses in $37 \%$ of patients [31]. These studies suggest an improvement in therapeutic efficacy with ${ }^{90} \mathrm{Y}$-labeled somatostatin analogues compared with ${ }^{111}$ In-labeled octreotide. The utility of this agent, however, may be limited by its potential to cause renal and hematologic toxicity.

Most recently, [Tyr3]octreotate labeled with ${ }^{177} \mathrm{Lu}$, a low-energy $\beta$ - and $\gamma$-particle emitter, was evaluated in a phase 1 study, with encouraging results. In one series, 131 patients with somatostatin receptor-positive, advanced neuroendocrine tumors received $\left[{ }^{177} \mathrm{Lu}\right.$-DOTA, Tyr3]octreotate administered every 6 to 10 weeks, to a final intended dose of 600 to 800 $\mathrm{mCi}$. Among patients with tumors that could be evaluated, there were 35 objective responses (28\%), three of which were complete [35]. [ ${ }^{177} \mathrm{Lu}-\mathrm{DOTA}$, Tyr3] octreotate has since been used in the treatment of more than 500 patients with neuroendocrine tumors. Efficacy results, reported for 310 patients, suggest an overall tumor response rate of up to $30 \%$ [36]. The development of somatostatin analogues with higher affinity for different somatostatin 
receptor subtypes has the potential to further enhance the therapeutic efficacy of this general treatment approach.

\section{Vascular Endothelial Growth Factor Pathway Inhibitors}

Neuroendocrine tumors are highly vascular, and overexpression of vascular endothelial growth factor (VEGF) has been observed in midgut carcinoid tumors and pancreatic endocrine tumors [37]. Similarly, expression of VEGF receptor (VEGFR) subtypes 1 and 2 has been observed in most carcinoid and pancreatic endocrine tumors, suggesting that binding of VEGF to its receptor may be involved in neuroendocrine tumorigenesis [38,39]. In a phase 2 trial, 44 patients with advanced or metastatic carcinoid tumors on a stable dose of octreotide were randomly assigned to receive bevacizumab, a humanized monoclonal antibody targeting VEGF, or pegylated IFN-a-2b (Table 2) [40••]. Four of 22 patients $(18 \%)$ treated with bevacizumab achieved confirmed radiographic partial responses as compared with none of the patients who received pegylated IFN-a-2b. After 18 weeks, 96\% of patients treated with bevacizumab remained progression-free, compared with $68 \%$ of patients treated with IFN-a-2b. These encouraging results have led to the development of an ongoing study, led by the Southwest Oncology Group, in which patients are randomly assigned to receive IFN or bevacizumab, with a primary end point of progression-free survival (Fig. 1A).

The small molecule tyrosine kinase inhibitor sorafenib, with activity against VEGFR-2 and platelet-derived growth factor receptor (PDGFR)- $\beta$, was evaluated in 50 patients with carcinoid and 43 patients with pancreatic neuroendocrine tumors. In a preliminary analysis, responses were observed in $7 \%$ of patients with carcinoid tumors and $11 \%$ of those with pancreatic neuroendocrine tumors (Table 2) [41]. Sunitinib, another small molecule tyrosine kinase inhibitor, has activity against VEGFR-1, -2 , and -3 ; PDGFR- $\alpha$ and $-\beta$; KIT; RET; FMS-like tyrosine kinase-3; and colony-stimulating factor receptor-1R. In a phase 2 study, 109 patients with advanced neuroendocrine tumors received repeated 6-week treatment cycles of sunitinib, administered at an oral dosage of $50 \mathrm{mg}$ once daily for 4 weeks, followed by 2 weeks off treatment (Table 2) [42••]. Partial responses were observed in $2 \%$ of the carcinoid cohort and $16 \%$ of the pancreatic neuroendocrine tumor cohort. An international randomized study to confirm the activity of sunitinib in pancreatic neuroendocrine tumors is ongoing (Fig. 1B).

\section{mTOR Inhibitors}

The mammalian target of rapamycin (mTOR) is a serine-threonine kinase that participates in the regulation of cell growth, proliferation, and apoptosis through modulation of the cell cycle [43]. mTOR modulates translation of key mRNAs of proteins required for cell cycle progression. Signaling through the phosphatidylinositol-3 kinase/AKT/mTOR pathway leads to increased translation of proteins regulating cell cycle progression and metabolism [44]. In cancer cells, aberrant activation of the pathway may occur through increased signaling through growth factor receptors, activating mutations/amplification of kinases, or loss of the tumor suppressor PTEN [45]. mTOR also mediates downstream signaling from 
several pathways, including the VEGF and insulin-like growth factor signaling implicated in neuroendocrine tumor growth.

Inhibitors of mTOR have shown promising early activity in several cancer types [46]. Sirolimus (rapamycin) and its derivatives are immunosuppressive macrolides that block mTOR. Two rapamycin derivatives were evaluated recently in neuroendocrine tumors: temsirolimus and everolimus (RAD001). Temsirolimus binds intracellularly to FK506binding protein-12 and creates a complex that inhibits protein kinase activity of mTOR (Table 2) [47]. Inhibition of mTOR prevents phosphorylation of key cell cycle control proteins, leading to $\mathrm{G} 1$ growth arrest. In a multicenter study, 37 patients with advanced progressive neuroendocrine tumors were treated with weekly intravenous temsirolimus. The intent-to-treat response rate for the cohort was 5.6\%. Outcomes were similar between patients with carcinoid tumors and those with pancreatic neuroendocrine tumors [47].

In another phase 2 study, 30 patients with carcinoid tumors and 30 with pancreatic neuroendocrine tumors were treated with a combination of the MTOR inhibitor RAD001 (everolimus; $5 \mathrm{mg} / \mathrm{d}$ ) and depot octreotide (30 mg every 4 weeks). The overall tumor response rate in evaluable patients was $17 \%$ in those with carcinoid tumors and $27 \%$ in those with pancreatic neuroendocrine tumors (Table 2) [48••]. Toxicity was generally manageable, and randomized trials to confirm the activity and safety of RAD001 in patients with neuroendocrine tumors are ongoing (Fig. 1C).

\section{Conclusions}

The recent development of newer somatostatin analogues with broader activity and higher binding affinity for somatostatin receptor subtypes may enhance symptom control in patients with neuroendocrine tumors refractory to current therapy. Peptide receptor radionuclide therapy holds further promise as an effective treatment modality. Over the past several years, important discoveries have improved our understanding of the molecular pathways important to neuroendocrine tumor development and growth. Preliminary results from clinical trials of VEGF pathway inhibitors and inhibitors of mTOR have been encouraging and suggest that these agents have the potential to inhibit tumor growth and, in some cases, result in tumor regression. Randomized trials are ongoing to confirm these results. Studies combining targeted therapies with each other, or with more traditional cytotoxic agents, are warranted.

\section{Acknowledgments}

This work was supported by National Cancer Institute grant P50 CA127003 (DF/HCC SPORE in Gastrointestinal Cancer). Additional support was provided by the Caring for Carcinoid Foundation, the Stephen and Caroline Kaufer fund for neuroendocrine tumor research, and Mr. Saul and Gitta Kurlat. We thank Lindy Morde for assistance in the preparation of the manuscript.

\section{References and Recommended Reading}

Papers of particular interest, published recently, have been highlighted as:

- Of importance 
•• Of major importance

1. Modlin I, Sandor A. An analysis of 8305 cases of carcinoid tumors. Cancer. 1997; 79:813-829. [PubMed: 9024720]

2. Yao JC, Hassan M, Phan A, et al. One hundred years after "carcinoid": epidemiology of and prognostic factors for neuroendocrine tumors in 35,825 cases in the United States. J Clin Oncol. 2008; 26:3063-3072. [PubMed: 18565894] In this updated analysis of the SEER database, the authors describe the incidence and prevalence of neuroendocrine tumors in the US population. The data suggest that carcinoid and other neuroendocrine tumors are more common than previously suspected.

3. Kloppel G, Perren A, Heitz PU. The gastroenteropancreatic neuroendocrine cell system and its tumors: the WHO classification. Ann N Y Acad Sci. 2004; 1014:13-27. [PubMed: 15153416]

4. Thorson A, Biorck G, Bjorkman G, et al. Malignant carcinoid of the small intestine with metastases to the liver, valvular disease on the right side of the heart (pulmonary stenosis and tricuspid regurgitation without septal defects), peripheral vasomotor symptoms, bronchoconstriction, and an unusual type of cyanosis. Am Heart J. 1954; 47:795-817. [PubMed: 13158264]

5. Sarmiento JM, Que FG. Hepatic surgery for metastases from neuroendocrine tumors. Surg Oncol Clin N Am. 2003; 12:231-242. [PubMed: 12735141]

6. Alsina AE, Bartus S, Hull D, et al. Liver transplant for metastatic neuroendocrine tumor. J Clin Gastroenterol. 1990; 12:533-537. [PubMed: 1699994]

7. Lang H, Oldhafer K, Weimann A, et al. Liver transplantation for metastatic neuroendocrine tumors. Ann Surg. 1997; 225:347-354. [PubMed: 9114792]

8. Le Treut YP, Delpero JR, Dousset B, et al. Results of liver transplantation in the treatment of metastatic neuroendocrine tumors. A 31-case French multicentric report. Ann Surg. 1997; 225:355364. [PubMed: 9114793]

9. Gupta S, Yao J, Ahrar K, et al. Hepatic artery embolization and chemoembolization for treatment of patients with metastatic carcinoid tumors: the MD Anderson experience. Cancer J. 2003; 9:261267. [PubMed: 12967136]

10. Loewe C, Schindl M, Cejna M, et al. Permanent transarterial embolization of neuroendocrine metastases of the liver using cyanoacrylate and lipiodol: assessment of mid- and long-term results. AJR Am J Roentgenol. 2003; 180:1379-1384. [PubMed: 12704055]

11. Moertel C, Johnson C, McKusick M, et al. The management of patients with advanced carcinoid tumors and islet cell carcinoma. Ann Int Med. 1994; 120:302-309. [PubMed: 8291824]

12. Rubin J, Ajani J, Schirmer W, et al. Octreotide acetate long-acting formulation versus open-label subcutaneous octreotide acetate in malignant carcinoid syndrome. J Clin Oncol. 1999; 17:600606. [PubMed: 10080605]

13. Kvols L, Moertel C, O'Connell M, et al. Treatment of the malignant carcinoid syndrome: evaluation of a long-acting somatostatin analog. N Engl J Med. 1986; 315:663-666. [PubMed: 2427948]

14. O'Toole D, Ducreux M, Bommelaer G, et al. Treatment of carcinoid syndrome: a prospective crossover evaluation of lanreotide versus octreotide in terms of efficacy, patient acceptability, and tolerance. Cancer. 2000; 88:770-776. [PubMed: 10679645]

15. Schmid HA, Schoeffter P. Functional activity of the multiligand analog SOM230 at human recombinant somatostatin receptor subtypes supports its usefulness in neuroendocrine tumors. Neuroendocrinology. 2004; 80(Suppl 1):47-50. [PubMed: 15477717]

16. Kvols L, Wiedenmann B, Oberg K, et al. Safety and efficacy of pasireotide (SOM230) in patients with metastatic carcinoid tumors refractory or resistant to octreotide LAR: results of a phase II study [abstract]. J Clin Oncol. 2006; 24:4082.

17. Frank M, Klose KJ, Wied M, et al. Combination therapy with octreotide and alpha-interferon: effect on tumor growth in metastatic endocrine gastroenteropancreatic tumors. Am J Gastroenterol. 1999; 94:1381-1387. [PubMed: 10235222]

18. Janson E, Oberg K. Long term management of the carcinoid syndrome: treatment with octreotide alone and in combination with alpha-interferon. Acta Oncol. 1993; 32:225-229. [PubMed: 7686765] 
19. Oberg K, Eriksson B. The role of interferons in the management of carcinoid tumors. Acta Oncol. 1991; 30:519-522. [PubMed: 1854509]

20. Sun W, Lipsitz S, Catalano P, et al. Phase II/III study of doxorubicin with fluorouracil compared with streptozocin with fluorouracil or dacarbazine in the treatment of advanced carcinoid tumors: Eastern Cooperative Oncology Group Study E1281. J Clin Oncol. 2005; 23:4897-4904. [PubMed: 16051944]

21. Moertel C, Lefkopoulo M, Lipsitz S, et al. Streptozocin-doxorubicin, streptozocin-fluorouracil, or chlorozotocin in the treatment of advanced islet-cell carcinoma. N Engl J Med. 1992; 326:519523. [PubMed: 1310159]

22. Kouvaraki M, Ajani J, Hoff P, et al. Fluorouracil, doxorubicin, and streptozocin in the treatment of patients with locally advanced and metastatic pancreatic endocrine carcinomas. J Clin Oncol. 2004; 22:4762-4771. [PubMed: 15570077]

23. Stevens MF, Hickman JA, Langdon SP, et al. Antitumor activity and pharmacokinetics in mice of 8-carbamoyl-3-methyl-imidazo[5,1-d]-1,2,3,5-tetrazin-4(3H)-one (CCRG 81045; M \& B 39831), a novel drug with potential as an alternative to dacarbazine. Cancer Res. 1987; 47:5846-5852. [PubMed: 3664486]

24. Kulke MH, Stuart K, Enzinger PC, et al. Phase II study of temozolomide and thalidomide in patients with metastatic neuroendocrine tumors. J Clin Oncol. 2006; 24:401-406. [PubMed: 16421420]

25. Kulke M, Stuart K, Earle C, et al. A phase II study of temozolomide and bevacizumab in patients with advanced neuroendocrine tumors [abstract]. J Clin Oncol. 2006; 24:4044. [PubMed: 16921067]

26. Ekeblad S, Sundin A, Janson ET, et al. Temozolomide as monotherapy is effective in treatment of advanced malignant neuroendocrine tumors. Clin Cancer Res. 2007; 13:2986-2991. [PubMed: 17505000]

27. Isacoff W, Moss R, Pecora A, et al. Temozolomide/capecitabine therapy for metastatic neuroendocrine tumors of the pancreas. A retrospective review [abstract]. J Clin Oncol. 2006; 24:14023.

28. Strosberg J, Choie J, Gardner N, et al. First-line treatment of metastatic endocrine carcinomas with capecitabine and temozolomide [abstract]. J Clin Oncol. 2008; 26:4612.

29. Krenning EP, Kwekkeboom DJ, Valkema R, et al. Peptide receptor radionuclide therapy. Ann N Y Acad Sci. 2004; 1014:234-245. [PubMed: 15153440]

30. Breeman WA, de Jong M, Kwekkeboom DJ, et al. Somatostatin receptor-mediated imaging and therapy: basic science, current knowledge, limitations and future perspectives. Eur J Nucl Med. 2001; 28:1421-1429. [PubMed: 11585303]

31. Teunissen JJ, Kwekkeboom DJ, de Jong M, et al. Endocrine tumours of the gastrointestinal tract. Peptide receptor radionuclide therapy. Best Pract Res Clin Gastroenterol. 2005; 19:595-616. [PubMed: 16183530]

32. Anthony LB, Woltering EA, Espenan GD, et al. Indium-111-pentetreotide prolongs survival in gastroenteropancreatic malignancies. Semin Nucl Med. 2002; 32:123-132. [PubMed: 11965607]

33. DeJong M, Valkema R, Jamar F, et al. Somatostatin-receptor targeted radionucleotide therapy of tumors: preclinical and clinical findings. Semin Nucl Med. 2002; 32:133-140. [PubMed: 11965608]

34. Virgolini I, Britton K, Buscombe J, et al. In- and Y-DOTA-lanreotide: results and implications of the MAURITIUS trial. Semin Nucl Med. 2002; 32:148-155. [PubMed: 11965610]

35. Kwekkeboom DJ, Teunissen JJ, Bakker WH, et al. Radio-labeled somatostatin analog $\left[{ }^{177} \mathrm{Lu}-\right.$ DOTA0, Tyr3] octreotate in patients with endocrine gastroenteropancreatic tumors. J Clin Oncol. 2005; 23:2754-2762. [PubMed: 15837990]

36. Kwekkeboom DJ, de Herder WW, Kam BL, et al. Treatment with the radiolabeled somatostatin analog [ ${ }^{177}$ Lu-DOTA 0,Tyr3]octreotate: toxicity, efficacy, and survival. J Clin Oncol. 2008; 26:2124-2130. [PubMed: 18445841]

37. Terris B, Scoazec J, Rubbia L. Expression of vascular endothelial growth factor in digestive neuroendocrine tumors. Histopathology. 1998; 32:133-138. [PubMed: 9543669] 
38. Christofori G, Naik P, Hanahan D. Vascular endothelial growth factor and its receptors, flt-1 and flk-1, are expressed in normal pancreatic islets and throughout islet cell tumorigenesis. Mol Endocrinol. 1995; 9:1760-1770. [PubMed: 8614412]

39. La Rosa S, Uccella S, Finzi G, et al. Localization of vascular endothelial growth factor and its receptors in digestive endocrine tumors: correlation with microvessel density and clinicopathologic features. Hum Pathol. 2003; 34:18-27. [PubMed: 12605362]

40. Yao JC, Phan A, Hoff PM, et al. Targeting vascular endothelial growth factor in advanced carcinoid tumor: a random assignment phase II study of depot octreotide with bevacizumab and pegylated interferon alpha-2b. J Clin Oncol. 2008; 26:1316-1323. [PubMed: 18323556] This prospective phase 2 study demonstrated activity associated with the VEGF inhibitor bevacizumab in patients with carcinoid tumor. The study was the basis for the development of a large confirmatory randomized trial.

41. Hobday TJ, Rubin J, Holen K, et al. MC044h, a phase II trial of sorafenib in patients (pts) with metastatic neuroendocrine tumors (NET): a Phase II Consortium (P2C) study [abstract]. J Clin Oncol. 2007; 25:4504.

42. Kulke MH, Lenz HJ, Meropol NJ, et al. Activity of sunitinib in patients with advanced neuroendocrine tumors. J Clin Oncol. 2008; 26:3403-3410. [PubMed: 18612155] This prospective phase 2 study demonstrated activity of the small molecule tyrosine kinase inhibitor sunitinib in neuroendocrine tumors. Sunitinib appeared to have greater activity in pancreatic neuroendocrine than carcinoid tumors.

43. Vignot S, Faivre S, Aguirre D, et al. mTOR-targeted therapy of cancer with rapamycin derivatives. Ann Oncol. 2005; 16:525-537. [PubMed: 15728109]

44. Podsypanina K, Lee RT, Politis C, et al. An inhibitor of mTOR reduces neoplasia and normalizes p70/S6 kinase activity in Pten+/- mice. Proc Natl Acad Sci U S A. 2001; 98:10320-10325. [PubMed: 11504907]

45. Wang L, Ignat A, Axiotis CA. Differential expression of the PTEN tumor suppressor protein in fetal and adult neuroendocrine tissues and tumors: progressive loss of PTEN expression in poorly differentiated neuroendocrine neoplasms. Appl Immunohistochem Mol Morphol. 2002; 10:139_ 146. [PubMed: 12051632]

46. Smolewski P. Recent developments in targeting the mammalian target of rapamycin (mTOR) kinase pathway. Anticancer Drugs. 2006; 17:487-494. [PubMed: 16702804]

47. Duran I, Kortmansky J, Singh D, et al. A phase II clinical and pharmacodynamic study of temsirolimus in advanced neuroendocrine carcinomas. Br J Cancer. 2006; 95:1148-1154. [PubMed: 17031397]

48. Yao JC, Phan AT, Chang DZ, et al. Efficacy of RAD001 (everolimus) and octreotide LAR in advanced low- to intermediate-grade neuroendocrine tumors: results of a phase II study. J Clin Oncol. 2008; 26:4311-4318. [PubMed: 18779618] The authors describe a prospective phase 2 study demonstrating activity associated with the mTOR inhibitor everolimus in carcinoid and pancreatic neuroendocrine tumors. The trial was the basis for two international phase 3 studies of everolimus in neuroendocrine tumors.

49. Ramanathan RK, Cnaan A, Hahn RG, et al. Phase II trial of dacarbazine (DTIC) in advanced pancreatic islet cell carcinoma. Study of the Eastern Cooperative Oncology Group-E6282. Ann Oncol. 2001; 12:1139-1143. [PubMed: 11583197] 


\section{A Southwest Oncology Group S0518/North American Intergroup:} phase 3 randomized comparison of octreotide + IFN- $\alpha$ vs octreotide + bevacizumab in carcinoid tumors

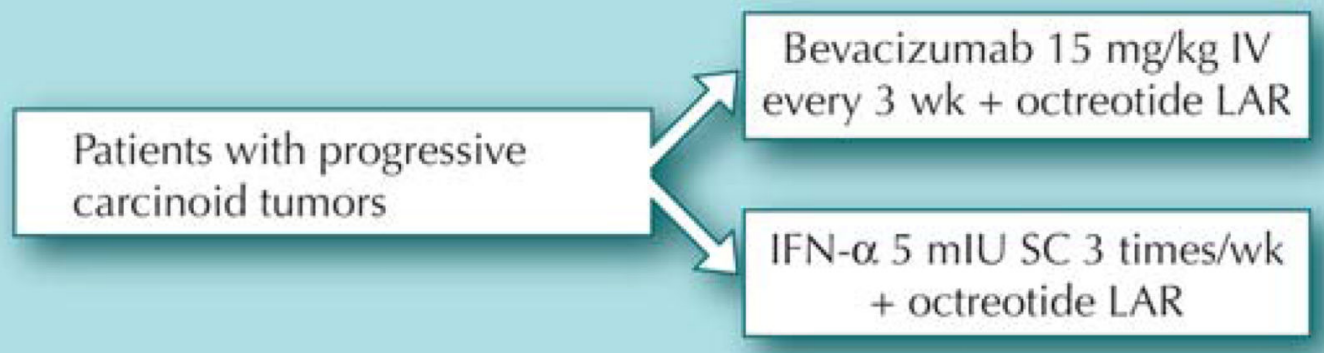

End point: PFS

B Phase 3 study of sunitinib compared with placebo in patients with pancreatic islet cell tumors (industry sponsored)

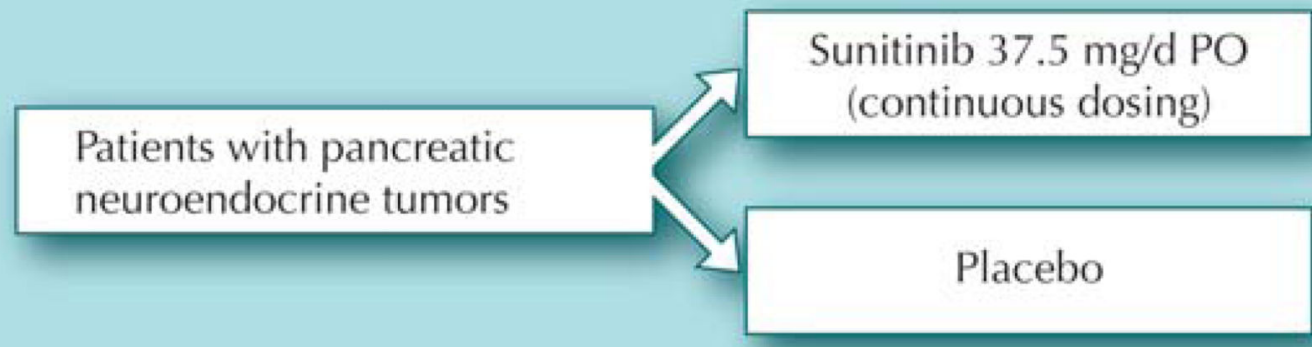

End point: PFS

C RADIANT studies in carcinoid and pancreatic neuroendocrine tumors (industry sponsored)

Patients with progressive carcinoid (RADIANT 2) or pancreatic neuroendocrine tumors (RADIANT 3)

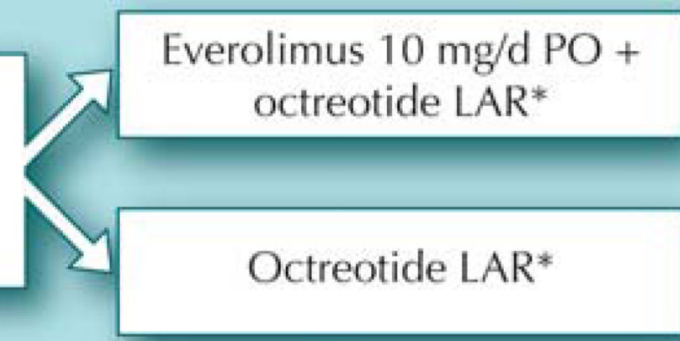

*Octreotide is not required for pancreatic neuroendocrine tumor patients.

Figure 1.

A-C, Selected ongoing randomized trials of novel antitumor therapies in neuroendocrine tumors. IFN_-interferon; IV_-intravenously; LAR_-long-acting release; PFS—progressionfree survival; PO_-orally; RADIANT_An Open Label, Stratified, Single-Arm Phase II Study of Everolimus in Patients With Advanced Pancreatic Neuroendocrine Tumor (NET) After Failure of Cytotoxic Chemotherapy; SC-subcutaneously. 
Table 1

Efficacy of streptozocin- or temozolomide-based regimens in metastatic pancreatic endocrine tumors

\begin{tabular}{|c|c|c|c|c|}
\hline Study & Regimen & Patients, $n$ & $\begin{array}{r}\text { Tumor response } \\
\text { rate, \% }\end{array}$ & $\begin{array}{c}\text { Median overall } \\
\text { survival, } \text { mo }\end{array}$ \\
\hline \multicolumn{5}{|l|}{ Prospective studies } \\
\hline \multirow[t]{3}{*}{ Moertel et al., 1992 [21] } & Chlorozotocin & 33 & 30 & 18.0 \\
\hline & Fluorouracil + streptozocin & 33 & 45 & 16.8 \\
\hline & Doxorubicin + streptozocin & 36 & 69 & 26.4 \\
\hline Ramanathan et al., 2001 [49] & Dacarbazine & 50 & 34 & 19.3 \\
\hline Kulke et al., 2006 [24] & Temozolomide + thalidomide & 11 & 45 & NR \\
\hline Kulke et al., 2006 [25] & Temozolomide + bevacizumab & 17 & 24 & NR \\
\hline \multicolumn{5}{|l|}{ Retrospective studies } \\
\hline Kouvaraki et al., 2004 [22] & Streptozocin + doxorubicin + fluorouracil & 84 & 39 & 37 \\
\hline Isacoff et al., 2006 [27] & Temozolomide + capecitabine & 17 & 59 & 8.9 \\
\hline Ekeblad et al., 2007 [26] & Temozolomide (single agent) & 12 & 8 & NR \\
\hline Strosberg et al., 2008 [28] & Temozolomide + capecitabine & 17 & 71 & NR \\
\hline
\end{tabular}

NR-not reported. 


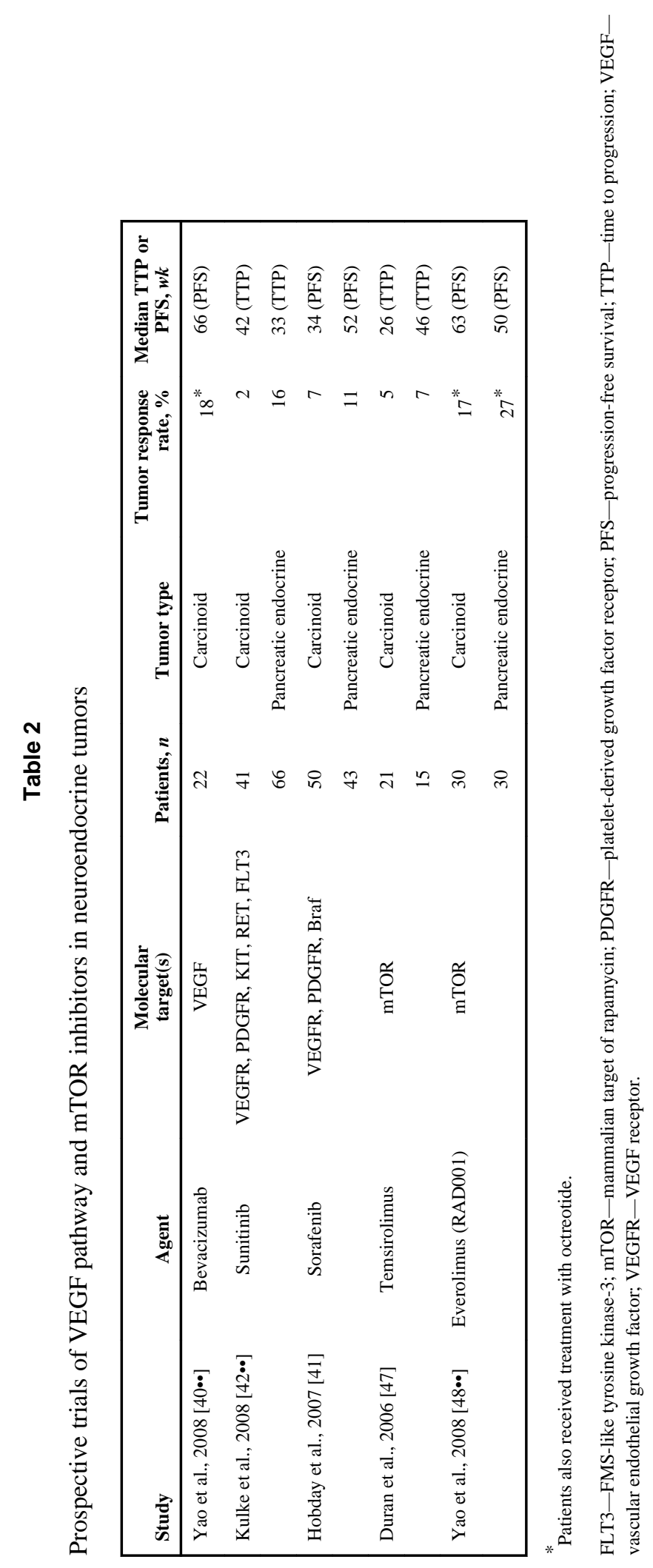

Curr Oncol Rep. Author manuscript; available in PMC 2014 May 21. 